\title{
Temperature and Strain Rate Dependence of Flow Stress in Severely Deformed Copper by Accumulative Roll Bonding
}

\author{
Takahiro Kunimine ${ }^{1, *}$, Naoki Takata ${ }^{2}$, Nobuhiro Tsuji ${ }^{3}$, \\ Toshiyuki Fujii $^{4}$, Masaharu Kato ${ }^{1}$ and Susumu Onaka ${ }^{1}$ \\ ${ }^{1}$ Department of Materials Science and Engineering, Tokyo Institute of Technology, Yokohama 226-8502, Japan \\ ${ }^{2}$ Department of Metallurgy and Ceramics Science, Tokyo Institute of Technology, Tokyo 152-8552, Japan \\ ${ }^{3}$ Department of Adaptive Machine Systems, Osaka University, Suita 565-0871, Japan \\ ${ }^{4}$ Department of Innovative and Engineered Materials, Tokyo Institute of Technology, Yokohama 226-8502, Japan
}

Tensile tests and strain rate jump tests have been carried out at low temperatures $(77 \mathrm{~K} \sim$ room temperature $(\mathrm{RT}))$ using pure $\mathrm{Cu}$ specimens that were severely deformed by accumulative roll bonding (ARB). The dependence of the flow stress on the temperature and the strain rate has been investigated and the strain rate sensitivity $m$ and its variation caused by the change in the ARB cycle $N$ are discussed. At RT, the strain rate sensitivity for $N \leq 4$ stays at about 0.005 . However, for $N \geq 5, m$ increases with increasing $N$ to become $\sim 0.018$ when $N=8$. The deformation mechanisms of the ARB processed $\mathrm{Cu}$ are discussed with the activation volume $V^{*}$. The temperature dependence of $V^{*}$ and its variation with increasing $N$ are also discussed. [doi:10.2320/matertrans.MD200809]

(Received July 22, 2008; Accepted October 6, 2008; Published November 19, 2008)

Keywords: copper, ultra-fine grained materials, accumulative roll bonding, strain rate sensitivity, activation volume, deformation mechanism

\section{Introduction}

The strain rate dependence of flow stress in ultra-fine grained (UFG) and nanocrystalline (NC) materials has been investigated extensively. ${ }^{1-4)}$ The higher strain rate dependence of the flow stress of UFG and NC materials compared with that of conventional materials has been reported at room temperature (RT). However, little is known about the temperature dependence of the flow stress in UFG materials prepared by severe plastic deformation (SPD) processes. ${ }^{1)}$ Although models to describe characteristic mechanical properties of UFG materials have been proposed, ${ }^{3-7)}$ experimental studies are still necessary to discuss the deformation mechanisms.

The accumulative roll bonding (ARB) is one of the SPD processes. ${ }^{8}$ ) This process provides homogeneous nanostructures and large bulky metallic materials. ${ }^{9)}$ In the present study, tensile tests and strain rate jump tests at low temperatures are made for ARB processed pure $\mathrm{Cu}$. The strain rate sensitivity as well as activation volume obtained by the strain rate jump tests are the indexes of the strain rate dependence of the flow stress. The variation of these strain rate parameters with the ARB cycles (i.e., the plastic strain applied by $\mathrm{ARB}$ ) together with the temperature dependence of strength are discussed.

\section{Experimental}

Polycrystalline copper of $99.99 \%$ purity was used as a starting material. After annealing at $873 \mathrm{~K}$ for $2 \mathrm{~h}$, copper sheets with an initial grain size of about $100 \mu \mathrm{m}$ were subjected to the ARB process at room temperature (RT) with lubrication. ${ }^{10)}$ The ARB process was carried out up to $N=8$ where $N$ is the number of ARB cycles. The equivalent strain for $N=8$ is about 6.4 in terms of the logarithmic strain. ${ }^{11)}$

*Graduate Student, Tokyo Institute of Technology
The ARB process initially induces microstructure with elongated grains to the rolling direction. The microstructure of copper at $N=4$ possesses the so-called lamellar boundary structure. ${ }^{12,13)}$ However, the microstructure for $N \geq 6$ consists of a mixture of relatively equiaxed and slightly elongated grains. ${ }^{12,13)}$ At $N=8$, the spacing of high-angle lamellar boundaries of elongated grains was about $370 \mathrm{~nm}$ in the present study.

Using an electro-discharging machine, tensile specimens with $10 \mathrm{~mm}$ gauge length and $3 \mathrm{~mm}$ gauge width were cut from the ARB processed sheets with $1 \mathrm{~mm}$ in thickness. Although the tensile axis is parallel to the ARB rolling direction for the 6 and 8-cycle specimens, other ARB processed specimens have the tensile axis parallel to the transverse direction of the sheets. Tensile specimens cut from the starting $\mathrm{Cu}$ polycrystal (the annealed $\mathrm{Cu}$ before ARB) were also prepared. Tensile tests were made with an Instrontype testing machine at $77,175 \mathrm{~K}$ (the melting point of methanol), 273 and $298 \mathrm{~K}$ within the accuracy of $\pm 2 \mathrm{~K}$.

Strain rate jump tests were conducted between the strain rates of $8.3 \times 10^{-5} \mathrm{~s}^{-1}$ and $8.3 \times 10^{-4} \mathrm{~s}^{-1}$. The strain rate sensitivity $m$ defined as

$$
m=\frac{\partial \ln \sigma}{\partial \ln \dot{\varepsilon}}=\frac{1}{\sigma} \frac{\partial \sigma}{\partial \ln \dot{\varepsilon}}
$$

is an index to evaluate the dependence of the flow stress $\sigma$ on the strain rate $\dot{\varepsilon}$. When a strain rate jump from $\dot{\varepsilon}_{1}$ to $\dot{\varepsilon}_{2}$ causes the change in the flow stress from $\sigma_{1}$ to $\sigma_{2}$ during tensile tests, the strain rate sensitivity $m$ is given by

$$
m \approx \frac{\Delta \sigma}{\sigma \Delta \ln \dot{\varepsilon}} \approx \frac{\left(\sigma_{2}-\sigma_{1}\right)}{\left[\left(\sigma_{1}+\sigma_{2}\right) / 2\right] \ln \left(\dot{\varepsilon}_{2} / \dot{\varepsilon}_{1}\right)} .
$$

For comparison, tensile tests of $99.99 \%$ copper single crystals were also performed. The single crystals were grown by the Bridgman method. Tensile specimens with $10 \mathrm{~mm}$ gauge length and $3 \mathrm{~mm}$ width were cut from the single crystals. The tensile axis of the specimens was chosen so that 


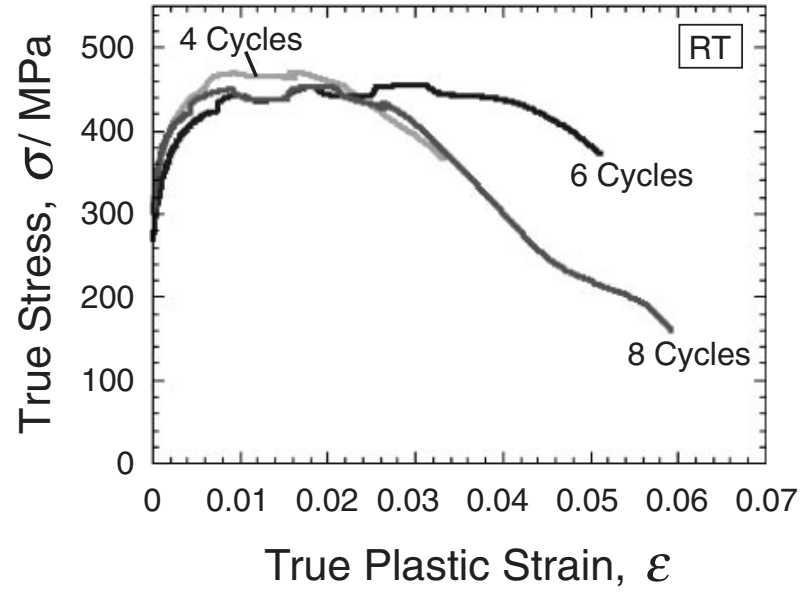

Fig. 1 The true stress-true strain $(\sigma-\varepsilon)$ curves of the specimens ARB processed by 4,6 and 8 cycles at RT. The steps on the $\sigma-\varepsilon$ curves are the changes in the flow stress caused by the strain rate jump tests between $8.3 \times 10^{-5} \mathrm{~s}^{-1}$ and $8.3 \times 10^{-4} \mathrm{~s}^{-1}$.

the initial plastic deformation occurs by single slip. The strain rate jump tests were carried out at 77,175 and $298 \mathrm{~K}$. The change in the strain rate sensitivity $m$ caused by work hardening was measured during tensile deformation.

\section{Results}

\subsection{Stress-strain curves at room temperature}

Figure 1 shows the true stress-true strain $(\sigma-\varepsilon)$ curves of specimens ARB processed by 4, 6 and 8 cycles at RT, which were simply converted from the nominal stress-nominal strain curves. Since uniform deformation is assumed to obtain true stress and true strain, the curves after macroscopic necking are not correct, strictly speaking. Although the tensile strength of the starting polycrystal before ARB was about $220 \mathrm{MPa}$, it increased with increasing the number of ARB cycles and nearly saturated $(450 \mathrm{MPa})$ even at $N=4$. This saturation stress is slightly higher than $400 \mathrm{MPa}$ reported in a previous study. ${ }^{13)}$ Another change in mechanical properties caused by ARB is the increase in ductility after 4 cycles: nearly twice larger at $N=8$ compared with that at $N=4$. A similar increase in ductility with increasing the number of ARB cycles or ECAP passes has also been found in previous studies. ${ }^{13-16)}$

The changes in the flow stress $\Delta \sigma$ associated with the sudden change of strain rate by a factor of ten amounted to $9 \mathrm{MPa}$ at $N=4$ and $18 \mathrm{MPa}$ at $N=8$. Generally speaking, $\Delta \sigma$ increased with increasing the number of ARB cycles.

\subsection{Strain rate sensitivity at room temperature}

The results of the strain rate jump tests for (1) the starting polycrystals (the annealed $\mathrm{Cu}$ before ARB), (2) the ARB processed specimens and (3) the single crystal specimens are summarized in Fig. 2 as the relationship between the strain rate sensitivity $m$ and the flow stress that were converted into the true shear stress $\tau$. We have evaluated the true shear stress $\tau$ from the true tensile stress $\sigma$ by using the Taylor factor $M_{\mathrm{T}}=3.06$ for (1) the starting polycrystals and (2) the ARB processed specimens, or by

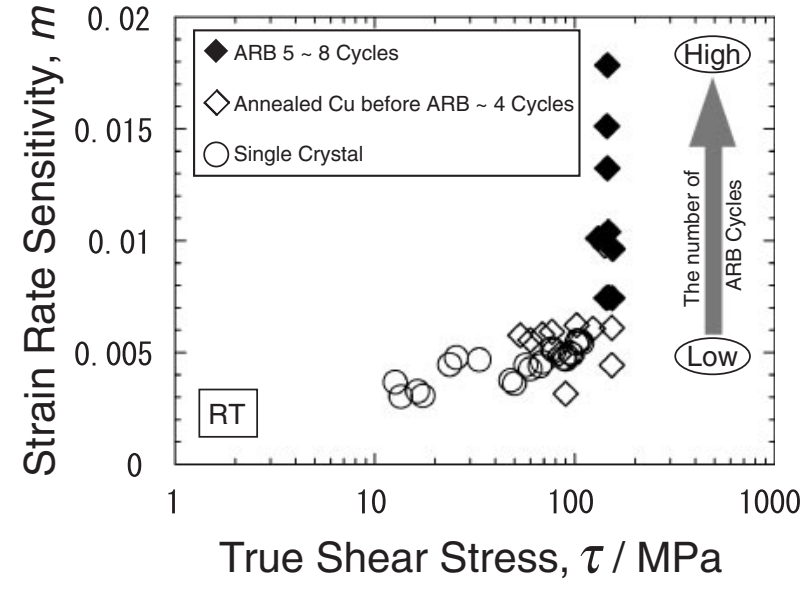

Fig. 2 The relationship between the strain rate sensitivity $m$ and the true shear stress $\tau$ at RT for the conventional polycrystals (the annealed $\mathrm{Cu}$ before ARB), the specimens ARB processed by various cycles and the single crystal specimens.

using the Schmid factor of 0.5 for (3) the single crystal specimens.

In Fig. 2, the data points shown by circles are the results for the single crystals, which indicate the variation of $m$ due to the increase in the true shear stress $\tau$ (work hardening during tensile deformation). Although $\tau$ increases from about 10 to $100 \mathrm{MPa}$ during the work hardening, the strain rate sensitivity $m$ does not change significantly and stays between 0.003 and 0.005 . These values are in agreement with previously reported values for work hardened $\mathrm{Cu}$ single crystals. ${ }^{17}$

On the other hand, the data points shown by open and closed diamonds in Fig. 2 are the results for the starting polycrystals and ARB processed specimens. The $m-\tau$ relations for the starting polycrystals and ARB processed specimens with $N \leq 4$ are similar to that for the single crystals. However, the higher-cycle ARB processes with $N \geq$ 5 markedly increase the $m$ value as shown by closed diamonds and $m$ reaches about 0.018 when $N=8$. The tensile strength of the ARB processed specimens with $N \geq 4$ was nearly the same as shown in Fig. 1, i.e., the large increase in $m$ and the almost constant $\tau$ are found for the higher-cycle ARB processed specimens.

The Cottrell-Stokes (CS) law states that the ratio $(\Delta \sigma / \sigma)$ is constant for various $\sigma$ when $\Delta \ln \dot{\varepsilon}$ is fixed. ${ }^{17-19)}$ Hence, from eq. (2), the strain rate sensitivity $m$ should be unchanged during plastic deformation if the CS law is obeyed. The validity of the CS law in f.c.c. single crystals during work hardening has been discussed in previous studies. ${ }^{17,20,21)}$ In contrast, the ARB processed $\mathrm{Cu}$ at $N=8$ shows the value of $m$ reaching 0.018 . Such high values of $m$ about 0.02 have been also reported for UFG $\mathrm{Cu}$ processed by ECAP. ${ }^{1-4)}$ In fact, it is known that nanocrystalline $\mathrm{Cu}$ and $\mathrm{Ni}$ show high values of $m$ such as $0.036^{22)}$ and $0.019 .^{6}$ )

\subsection{Stress-strain curves and strain rate sensitivity at low temperatures}

Figure 3 shows the temperature dependence of the true stress-true strain $(\sigma-\varepsilon)$ curves of ARB processed specimens 


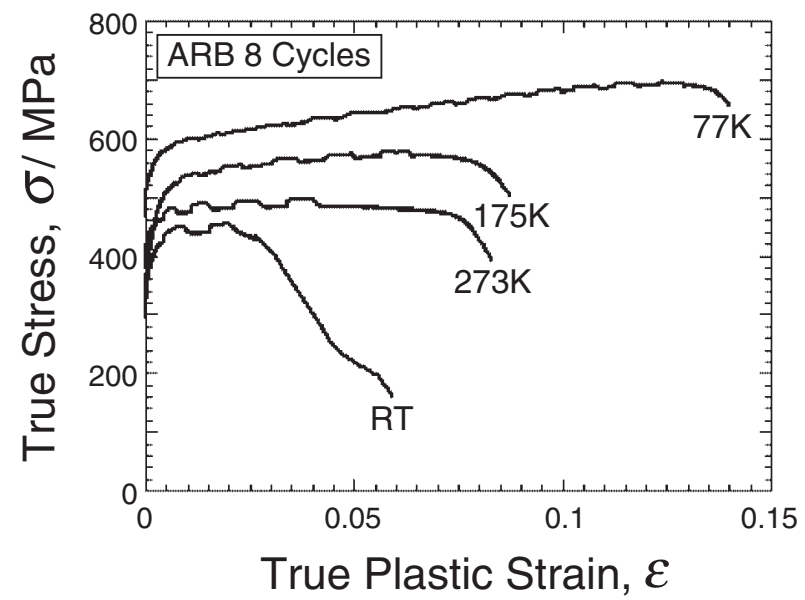

Fig. 3 The true stress-true strain $(\sigma-\varepsilon)$ curves of the 8-cycle ARB specimens tested at $77,175,273 \mathrm{~K}$ and $\mathrm{RT}$.

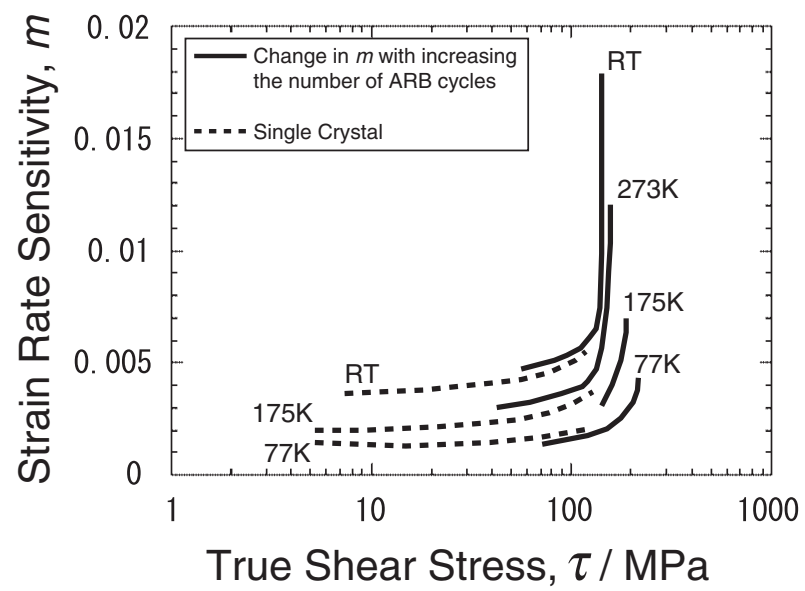

Fig. 4 The relationship between the strain rate sensitivity $m$ and the true shear stress $\tau$ for the usual polycrystals (the annealed $\mathrm{Cu}$ before ARB), the specimens ARB processed by various cycles and the single crystal specimens tensile-tested at 77, 175, $273 \mathrm{~K}$ and RT.

by 8 cycles. With decreasing the test temperature $T$ from RT to $77 \mathrm{~K}$, both strength and ductility increase. However, with decreasing $T, \Delta \sigma$ caused by the strain rate jump test decreases. As a result, the strain rate sensitivity $m$ given by eq. (2) decreases with decreasing $T$. Figure 4 shows the $m-\tau$ relations at various temperatures. As expected, the $m-\tau$ curve moves towards lower $m$ and higher $\tau$ with decreasing $T$, as observed earlier in $\mathrm{Cu}$ single crystals. ${ }^{17)}$ The higher-cycle ARB processes with $N \geq 5$ increase $m$ at 273 and $175 \mathrm{~K}$. However, this effect of $N$ on $m$ is less pronounced at $77 \mathrm{~K}$.

Figure 5 shows the relationships between $m$ and $T$. The data in this figure were obtained by plotting $m$ measured at a certain strain: $\varepsilon=0.01$ for the ARB processed specimens, $\varepsilon=0.15$ for the annealed $\mathrm{Cu}$ before $\mathrm{ARB}$ and $\varepsilon=$ 0.02 for the single crystals. We find that $m$ decreases with decreasing $T$ for all the specimens. We can see that the dependence of the $m$ value on the number $N$ of the ARB cycles decreases as temperature lowers. A similar decrease in $m$ with decreasing temperature between 273 and $523 \mathrm{~K}$ has also been found for aluminium processed by severe plastic deformation. ${ }^{16,23)}$

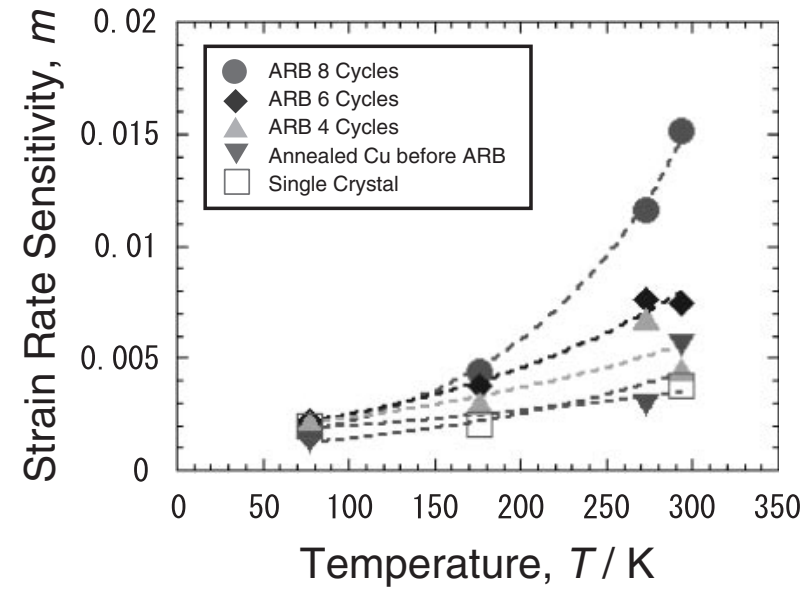

Fig. 5 The relationship between $m$ and $T$. The curves in this figure giving the $T$ dependence of $m$ are obtained by considering $m$ at certain strains, i.e., $\varepsilon=0.01$ for the specimens ARB processed by 4,6 and 8 cycles, $\varepsilon=0.15$ for the annealed $\mathrm{Cu}$ before $\mathrm{ARB}$ and $\varepsilon=0.02$ for the single crystals.

\section{Discussion}

\subsection{Activation volume at room temperature}

The rate equation to describe thermally activated plastic flow is given by

$$
\dot{\gamma}=\dot{\gamma}_{0} \exp \left(-G^{*} / k T\right)
$$

where $\dot{\gamma}$ is the shear strain rate, $\dot{\gamma}_{0}$ the pre-exponential constant, $k$ the Boltzmann constant and $G^{*}$ the activation energy. ${ }^{24,25)}$ The activation volume $V^{*}$ is given by ${ }^{24)}$

$$
V^{*}=-\left(\partial G^{*} / \partial \tau\right)=k T(\partial \ln \dot{\gamma} / \partial \tau),
$$

where $\tau$ is the applied shear stress. Then, from eqs. (1) and (4), $V^{*}$ is written as a function of $m$ :

$$
V^{*}=M_{\mathrm{T}} \frac{k T \partial \ln \dot{\varepsilon}}{\partial \sigma}=M_{\mathrm{T}} \frac{k T}{m \sigma} .
$$

Although the strain rate sensitivity $m$ itself is a phenomenological parameter obtained by mechanical testing, $m$ can be related to the activation volume $V^{*}$ by eq. (5).

The activation volume $V^{*}$ is also a value connected with the geometry of the activation process. Let us suppose that many short-range obstacles to the motion of dislocations are dispersed in materials and the activation process of plastic deformation is the interaction between a dislocation and a short-range obstacle. Then, the activation volume $V^{*}$ is written as

$$
V^{*}=L d^{*} b
$$

Where $L$ is the dislocation length which takes part in a local activation event, $d^{*}$ the average activation distance and $b$ the magnitude of the Burgers vector. The product $L d^{*}$ is the activation area swept by a dislocation during the activation event.

Figure 6 shows the relationships between the true shear stress $\tau$ and the activation volume $V^{*}$ (calculated from eq. (5)) for the specimens tested at RT. Since $m$ was almost constant during work hardening of the single crystals, it is natural that the calculated $V^{*}$ of the single crystals is inversely proportional to the true shear stress $\tau{ }^{26)}$ Similar 


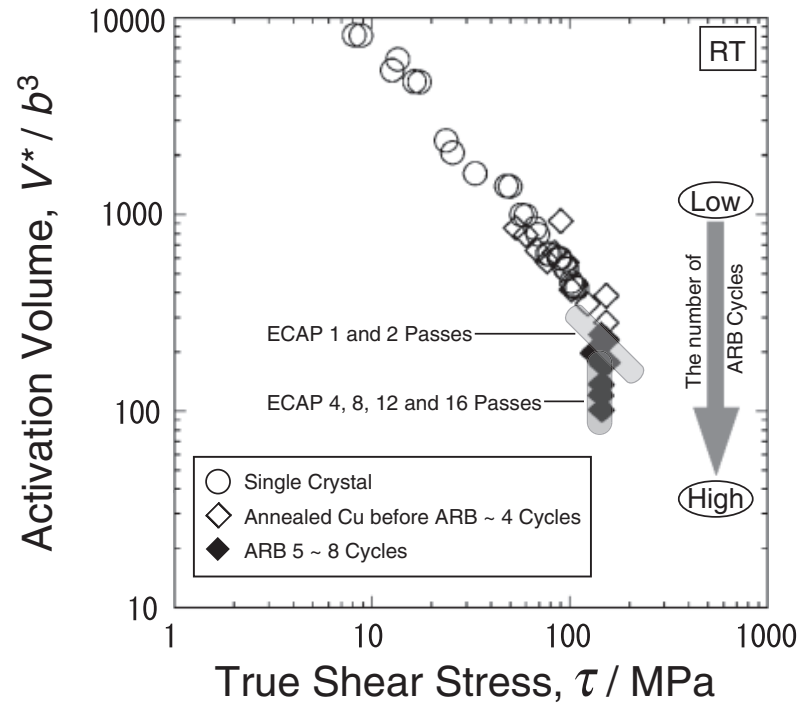

Fig. 6 The activation volume $V^{*}$ as a function of the true shear stress $\tau$ at RT. The previous results given by Dalla Torre et $_{\text {al. }}{ }^{3,4)}$ for ECAPed Cu are also shown by the hatched regions in this figure.

$V^{*}-\tau$ relation is also found for the starting polycrystals and the ARB processed specimens with $N \leq 4$. As shown in the log-log plot of Fig. 6 , these data points lie on a single line with a slope of -1 .

To understand the $V^{*} \propto 1 / \tau$ relation, let us consider the Bailey-Hirsch equation

$$
\tau=\alpha \mu b \sqrt{\rho},
$$

where $\rho$ is the density of forest dislocations, $\alpha$ a constant (0.3-0.6) and $\mu$ the shear modulus. ${ }^{27)}$ When the forest dislocations are the thermal obstacles to the motion of primary dislocations, the spacing between the obstacles $L$ can be considered as the dislocation length assigned to one activation event. Therefore, we have

$$
L \approx 1 / \sqrt{\rho} .
$$

From eqs. (6), (7) and (8) and assuming $d^{*} \approx b$, we indeed have the $V^{*} \propto 1 / \tau$ relation of the form

$$
V^{*} \approx \alpha \mu b^{3} / \tau \text {. }
$$

On the other hand, the obvious deviation from the $V^{*} \propto$ $1 / \tau$ relation with increasing number of ARB cycles must be explained in a different manner. Dalla Torre et al. have also reported such a decrease in $V^{*}$ for $\mathrm{Cu}$ severely processed by ECAP. ${ }^{3,4)}$ For comparison, their data are included in Fig. 6. The anomalous decrease in $V^{*}$ appears to be common to $\mathrm{Cu}$ specimens that were severely deformed by ARB or by ECAP. This point will be discussed below.

\subsection{The temperature dependence of the activation volume}

Using the results shown in Fig. 5 and eq. (5), we have obtained the temperature $T$ dependence of the activation volume $V^{*}$. Figure 7 shows the $V^{*}-T$ relations for various specimens. For the starting polycrystals and ARB processed specimens at $N=4, V^{*}$ decreases with decreasing $T$, as in the case of coarse-grained polycrystals. ${ }^{5)}$ Generally speaking, flow stress is lower and work hardening is less significant at

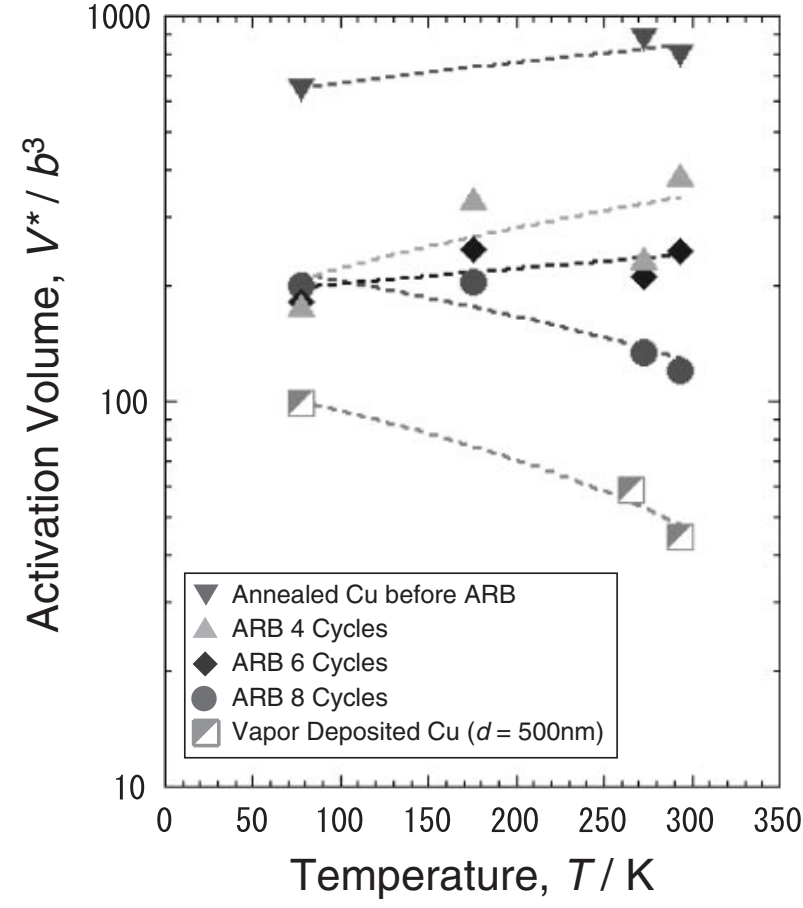

Fig. 7 The activation volume $V^{*}$ as a function of the temperature $T$. The previous results for vapor deposited $\mathrm{Cu}(d=500 \mathrm{~nm})$ shown in the literature $^{5)}$ are also indicated.

higher temperatures. This means that some of the obstacles to the motion of dislocations are not effective and $L$ is larger at higher temperatures. Moreover, the activation distance $d^{*}$ is usually an increasing function of temperature (decreasing function of stress). Since both $L$ and $d^{*}$ become larger as temperature becomes higher, the experimental result that $V^{*}$ is an increasing function of $T$ can be understood naturally.

However, Fig. 7 shows that the $T$ dependence of $V^{*}$ starts to change with increasing the number of ARB cycles $N$. For the ARB processed specimens at $N=6, V^{*}$ is almost independent of $T$ and, then, $V^{*}$ certainly increases with decreasing $T$ in the ARB processed specimens at $N=8$. The same $T$ dependence of $V^{*}$ also has been observed in vapor deposited $\mathrm{Cu}(d=500 \mathrm{~nm}),{ }^{5)}$ as shown in Fig. 7. Judging from the fact that Wang et al. reported the same temperature dependence of $V^{*}$ for $\left.\mathrm{NC} \mathrm{Ni}(d=30 \mathrm{~nm}){ }^{6}\right)$ it appears that the change in the temperature dependence of $V^{*}$ with decrease in the grain size is a universal phenomenon in f.c.c. UFG metals. As shown in Fig. 7, the transition in the $T$ dependence of $V^{*}$ occurs in the present ARB processed $\mathrm{Cu}$ at $N=6$. This critical number $N=6$ of the ARB cycles coincides reasonably with the onset of the anomalous increase of $m$ in Fig. 2 and with the start of the anomalous decrease in $V^{*}$ in Fig. 6.

To discuss this transition, it is necessary to consider changes in microstructure with increasing the ARB cycle. Grain refinement occurs markedly by the initial ARB process up to $N=2 .{ }^{28)}$ Afterward, the spacing of high-angle lamellar boundaries slightly decreases with increasing $N$ and approaches to a constant size, about $350 \mathrm{~nm}$ when $N \geq 4 .^{28)}$ In addition to the formation of lamellar boundary structure, the ARB process initially induces deformation microstructure consisting of low-angle boundaries. ${ }^{28)}$ However, the fraction 
(a) Low Temperature

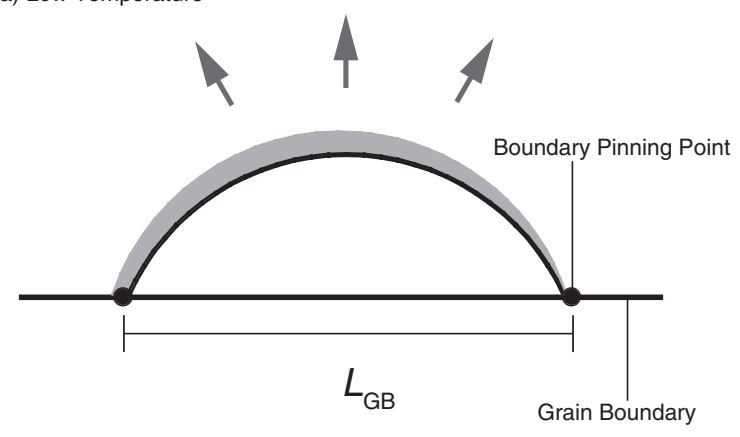

(b) Room Temperature

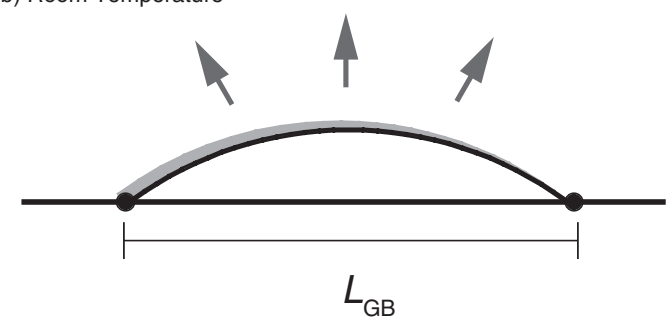

Fig. 8 The schematic illustration showing the shapes of bowing-out dislocations emitted from grain-boundary sources between pinning obstacles (a) at low temperature, (b) at room temperature.

$f_{\mathrm{HAGB}}$ of high-angle boundaries in the microstructure increases when $N \geq 2$. The $N$ dependence of $f_{\mathrm{HAGB}}$ shown in previous studies for $\mathrm{Cu}$ is $f_{\mathrm{HAGB}} \approx 30 \%$ at $N=2,{ }^{28)}$ $f_{\mathrm{HAGB}} \approx 55 \%$ at $N=6^{28)}$ and $f_{\mathrm{HAGB}} \approx 60 \%$ at $N=8 .^{29)}$ When $N \geq 6$, both the ultra-fine grains and the large fraction of high-angle boundaries are developed in the ARB processed $\mathrm{Cu}{ }^{28,29)}$ An explanation of the transition in the $T$ dependence of $V^{*}$ is possible if we assume that the ratecontrolling deformation mechanism changes, as will be discussed below, when such characteristic microstructures develop in $\mathrm{Cu}$.

Instead of the interaction between dislocations and the intra-grain obstacles, Kato et al. have proposed a dislocation bow-out model to explain mechanical properties of UFG materials. ${ }^{7)}$ In this model, a glide dislocation is considered to be nucleated at grain-boundary sources and bow out between two pinning points on the boundary. Plastic deformation occurs when a dislocation takes an unstable shape under the applied stress. The dislocation bow-out between pinning points on a grain boundary is illustrated in Fig. 8. The boundary pinning points are most probably short-range obstacles for the detachment of dislocations from the boundary, as pointed out by Wang et al. ${ }^{6)}$ Then, a thermal activation process can operate and a smaller dislocation bowout is sufficient to thermally overcome the obstacles as temperature becomes higher. As a result, as shown in Fig. 8, the associated activation area (shaded area) becomes smaller at a higher temperature. Therefore, when the thermally activated escape of a dislocation from boundary pinning points is rate-controlling, the anomalous $V^{*}$ dependence on $T$ can be explained at least qualitatively.

It is now generally believed that for plastic deformation of UFG and NC materials, grain-boundary dislocation sources becomes more important than in-grain sources as grain size becomes smaller. ${ }^{30,31)}$ In this respect, the present study may be regarded as an attempt to find the transition of the deformation mechanism. Further experimental and theoretical studies will reveal whether or not the present discussion, analysis and assumptions are indeed effective and reasonable.

\section{Conclusions}

The most important findings of this study on copper after severe plastic deformation by ARB are:

(1) At RT, the values of the strain rate sensitivity $m$ for the single crystals and the polycrystals before ARB are about 0.005. The low-cycle ARB processes with $N \leq 4$ do not affect $m$. However, the high-cycle ARB processes with $N \geq 5$ increase the values of $m$ to be 0.018 when $N=8$.

(2) The strain rate sensitivity $m$ monotonically decreases with decreasing the temperature from room temperature. Although the increase in $m$ caused by the increase in the ARB cycle is clear at RT when $N \geq 5$, this effect of $N$ on $m$ is less pronounced at $77 \mathrm{~K}$.

(3) The flow stress and temperature dependence of the activation volume $V^{*}$ suggests that the transition of deformation mechanisms in the ARB processed specimens occurs at the ARB cycle $N=6$.

\section{Acknowledgements}

This research was supported by a Grant-in-Aid for Scientific Research on Priority Areas "Giant straining process for advanced materials containing ultra-high density lattice defects" (18062002) by the Ministry of Education, Culture, Sports, Science and Technology of Japan.

\section{REFERENCES}

1) Y. M. Wang and E. Ma: Appl. Phys. Lett. 83 (2003) 3165-3167.

2) Q. Wei, S. Cheng, K. T. Ramesh and E. Ma: Mater. Sci. Eng. A 381 (2004) 71-79.

3) F. H. Dalla Torre, E. V. Pereloma and C. H. J. Davies: Scr. Mater. 51 (2004) 367-371.

4) F. H. Dalla Torre, E. V. Pereloma and C. H. J. Davies: Acta Mater. 54 (2006) 1135-1146.

5) H. Conrad: Mater. Sci. Eng. A 341 (2003) 216-228.

6) Y. M. Wang, A. V. Hamza and E. Ma: Acta Mater. 54 (2006) 27152726.

7) M. Kato, T. Fujii and S. Onaka: Mater. Trans. 49 (2008) 1278-1283.

8) Y. Saito, N. Tsuji, H. Utsunomiya, T. Sakai and H. G. Hong: Scr. Mater. 39 (1998) 1221-1227.

9) B. L. Li, N. Tsuji and N. Kamikawa: Mater. Sci. Eng. A 423 (2006) 331-342.

10) N. Kamikawa, T. Sakai and N. Tsuji: Acta Mater. 55 (2007) 58735888.

11) B. S. Altan, I. Miskioglu, G. Purcek, R. R. Mulyukov and R. Artan (eds.): Severe Plastic Deformation, Towards Bulk Production of Nanostructured Materials, (NOVA Science Publishers, New York, 2006) pp. 543-564.

12) N. Takata, K. Yamada, K. Ikeda, F. Yoshida, H. Nakashima and N. Tsuji: Mater. Trans. 48 (2007) 2043-2048.

13) N. Takata, S.-H. Lee, C.-Y. Lim, S.-S. Kim and N. Tsuji: J. Nanosci. Nanotechno. 7 (2007) 1-5.

14) H.-W. Kim, S.-B. Kang, N. Tsuji and Y. Minamino: Acta Mater. 53 (2005) 1737-1749.

15) H. W. Höppel, J. May and M. Göken: Adv. Eng. Mater. 6 (2004) 781-784.

16) H. W. Höppel, J. May, P. Eisenlohr and M. Göken: Z. Metallkd. 96 (2005) 566-571. 
17) W. Bochniak: Acta Metall. Mater. 43 (1995) 225-233.

18) A. H. Cottrell and R. J. Stokes: Proc. Roy. Soc. A 233 (1955) 17-34.

19) M. A. Adams and A. H. Cottrell: Philos. Mag. 46 (1955) 1187-1193.

20) W. Bochniak: Acta Metall. Mater. 41 (1993) 3133-3140.

21) S. Saimoto and H. Sang: Acta Metall. 31 (1983) 1873-1881.

22) L. Lu, R. Schwaiger, Z. W. Shan, M. Dao, K. Lu and S. Suresh: Acta Mater. 53 (2005) 2169-2179.

23) J. May, H. W. Höppel and M. Göken: Scr. Mater. 53 (2005) 189-194.

24) G. B. Gibbs: Phys. Stat. Sol. 10 (1965) 507-512.

25) B. de Meester, C. Yin, M. Doner and H. Conrad: Rate processes in plastic deformation of materials, (ASM, Metals Park, Ohio, 1975) pp. 175-226.

26) K. Saitou and T. Hikage: J. Japan Inst. Metals 49 (1985) 344-351.

27) H. Mughrabi: Constitutive Equations in Plasticity, (M. I. T. Press, Cambridge, Mass., 1975) pp. 199-250.

28) B. L. Li, N. Shigeiri, N. Tsuji and Y. Minamino: Mater. Sci. Forum 503-504 (2006) 615-620.

29) N. Shigeiri: Master Thesis, Osaka University (2006).

30) S. Cheng, J. A. Spencer and W. W. Milligan: Acta Mater. 51 (2003) $4505-4518$.

31) Y. J. Li, X. H. Zeng and W. Blum: Acta Mater. 52 (2004) 50095018. 